\section{JTI}

JOURNAL OF

TRAUMA AND INJURY

\title{
PARK Formula Can Replace "Guide to Medical Certificate” Published by Ko- rean Medical Association in Deciding the Treatment Duration
}

\author{
Chan Yong Park, M.D. ${ }^{1}$, Kwang Hee Yeo, M.D. ${ }^{2}$, Sora Ahn, M.D. ${ }^{1}$ \\ ${ }^{1}$ Department of Trauma Surgery, Wonkwang University Hospital, Iksan, Korea \\ ${ }^{2}$ Department of Trauma Surgery, Pusan National University Hospital, Busan, Korea
}

Received: March 4, 2018

Revised: April 10, 2018

Accepted: April 23, 2018

\section{Correspondence to}

Sora Ahn, M.D.

Department of Trauma Surgery, Wonkwang University Hospital, $895 \mathrm{Mu}-$ wang-ro, lksan 54538 , Korea

Tel: +82-63-859-0646

Fax: +82-63-855-2386

E-mail:sora46@hanmail.net
Purpose: Many doctors have difficulty in deciding the treatment duration in trauma patients to write in the casualty medical certificate. We tried to find a solution for this problem by using abbreviated injury scale (AIS).

Methods: A total of 39 patients treated in our regional trauma center who requested an author to write treatment duration on casualty medical certificate from January 2014 to April 2017 were included. And the treatment duration was decided based on the PARK Formula (AIS). PARK Formula $($ AIS $)=($ AIS $\times 2) \sim([$ AIS $\times 2]+2)$

Results: Among 39 patients included and 36 (92.3\%) had treatment duration on casualty medical certificate within the range of treatment duration calculated by PARK Formula (AIS). Compared to the PARK Formula (AIS), the mean value was 0.13 week $(0.90$ day) smaller. Comparing the treatment duration between Korean Medical Association (KMA) guideline and PARK Formula (AIS), only 22 patients (56.4\%) showed agreement. The mean value was 1.02 week (7.18 days) smaller in KMA guideline.

Conclusions: For the decision of the treatment duration in trauma patients, utilizing worldwide used AIS scoring system is very efficient. Using PARK Formula (AIS), doctors can document the treatment duration in the casualty medical certificate with ease. KMA should provide more practical 'treatment duration of each diagnosis in writing casualty medial certificate' for the doctors. We recommend PARK Formula (AIS) as a good alternative for KMA guide.

Keywords: Treatment duration; Casualty medical certificate; Abbreviated injury scale; Formula; Korean Medical Association 


\section{INTRODUCTION}

It is difficulty for many doctors to decide the treatment duration in the casualty medical certificate. The reason is that issuing medial certificate is not only just a medical practice, but also a source of economical and legal problem between the victim and offender [1]. To ease this problem, Korean Medical Association (KMA) published the first edition of 'guide to medical certificate' in 1996, and updated version was released in 2003 and 2015 [2-4]. However, in utilizing 'treatment duration of each diagnosis in writing casualty medial certificate' which is contained in guide to medial certificate 2015, there are a lot of situations requiring doctor's subjective decision due to the mixed description of diagnosis/operation name and the paucity of diagnosis. Also, it is difficult to find the difference in treatment duration according to the severity of the injury [4]. Therefore, we tried to figure out this problem using abbreviated injury scale (AIS) which is widely used in the field of traumatology. By using AIS which describes the whole body injury in detail, we can find the correct diagnosis easily, and this can guide doctors to decide the duration of treatment easily.

AIS is a globally used anatomy based injury scoring system developed by Association for the Advancement of Automotive Medicine (AAAM). The first edition of AIS was published in 1969 [5], and there were major revisions in 1976, 1980, 1985, 1990, 1998, 2005, 2008, and 2015 [6]. AIS classifies each part of the whole body according to the ordinary scale of 1 to 6 , which means that the higher the scale, the higher the severity of injury (Table 1).

Table 1. Abbreviated injury scale (AIS)

\begin{tabular}{|lll|}
\hline AIS code & \multicolumn{1}{c|}{ Injury } & \multicolumn{1}{c|}{ Example } \\
\hline 1 & Minor & Superficial laceration \\
2 & Moderate & Contusion of kidney \\
3 & Serious & Perforation of small bowel \\
4 & Severe & Proximal transection of pancreas \\
\hline 5 & Critical & Avulsion of spleen \\
6 & Maximum & Avulsion of liver \\
\hline
\end{tabular}

\section{METHODS}

Patients treated in our regional trauma center who requested an author to write the treatment duration on casualty medical certificate from January 2014 to April 2017 were included for this study. A total of 39 patients were included and the treatment duration was decided based on PARK Formula (AIS).

PARK Formula $($ AIS $)=($ AIS $\times 2) \sim([$ IIS $\times 2]+2)$

The PARK Formula (AIS) was derived by analyzing the correlation between treatment duration of liver injury in medical certificate guideline of KMA and AIS. With PARK Formula (AIS), treatment duration can be decided within a range of 3 weeks, and within each limitation, 1 week difference can be given according severity of injurymild, moderate, and severe.

\section{RESULTS}

Of the 39 patients, there were four neck injuries, five chest injuries, 29 abdominal injuries, and one lower extremity injury. The actual treatment duration of $36(92.3 \%)$ was within the range of the treatment duration calculated by PARK Formula (AIS). The other three patients, one patient required 1 week less treatment duration, and two patients required 2 weeks less treatment duration. Compared with PARK Formula (AIS), the actual treatment duration was 0.13 weeks ( 0.90 days) smaller. When comparing the results of the treatment duration of KMA guideline and PARK Formula (AIS), 22 patients (56.4\%) showed agreement. Among 17 discrepancies, two patients required 1 week, 11 patients required 2 weeks, four patients required 4 weeks less treatment duration in KMA guideline result (Table 2).

\section{DISCUSSION}

It is not unusual to have difficulty deciding the treatment duration for writing casualty medical certificates for trauma patients. The treatment duration can be different-in 


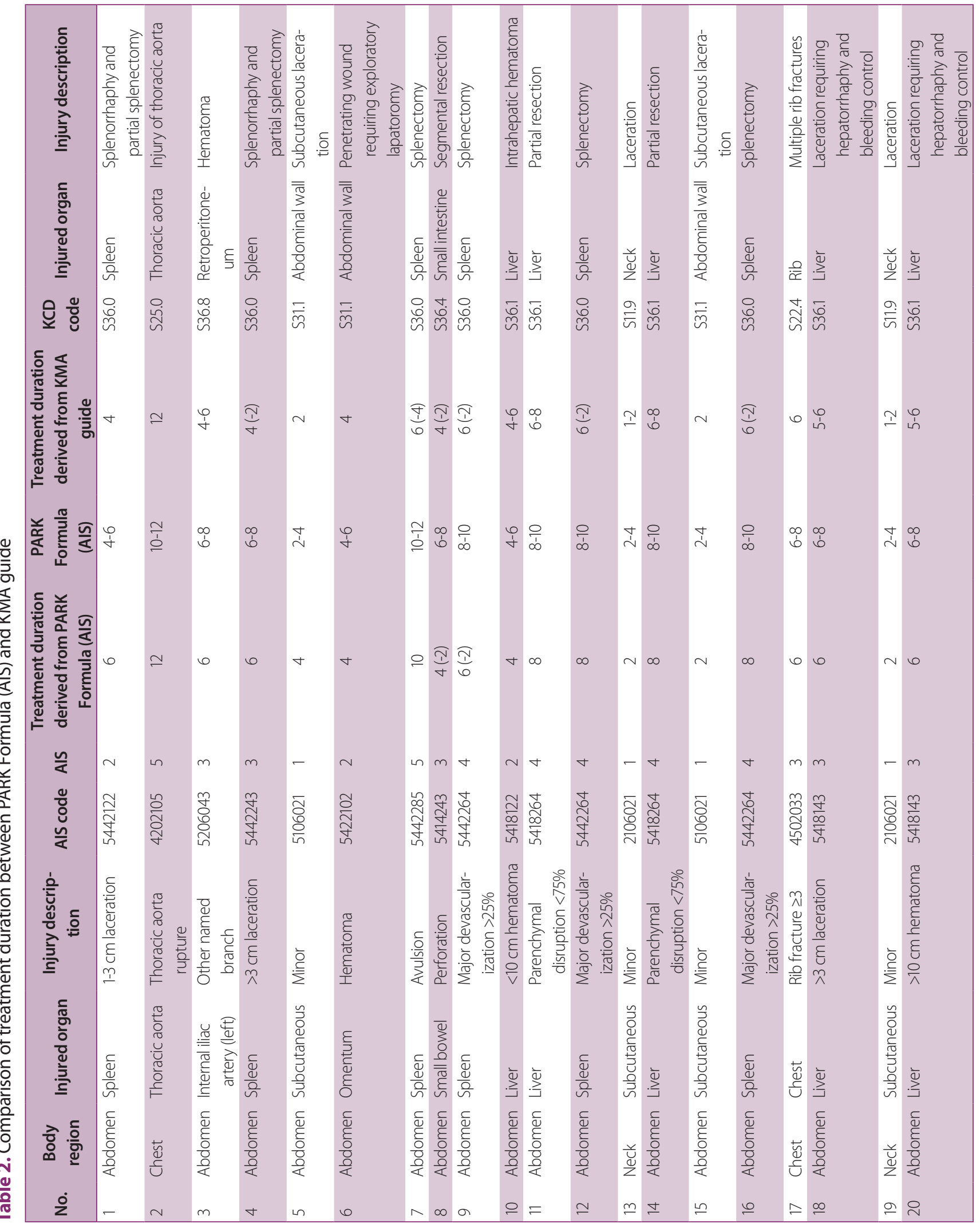




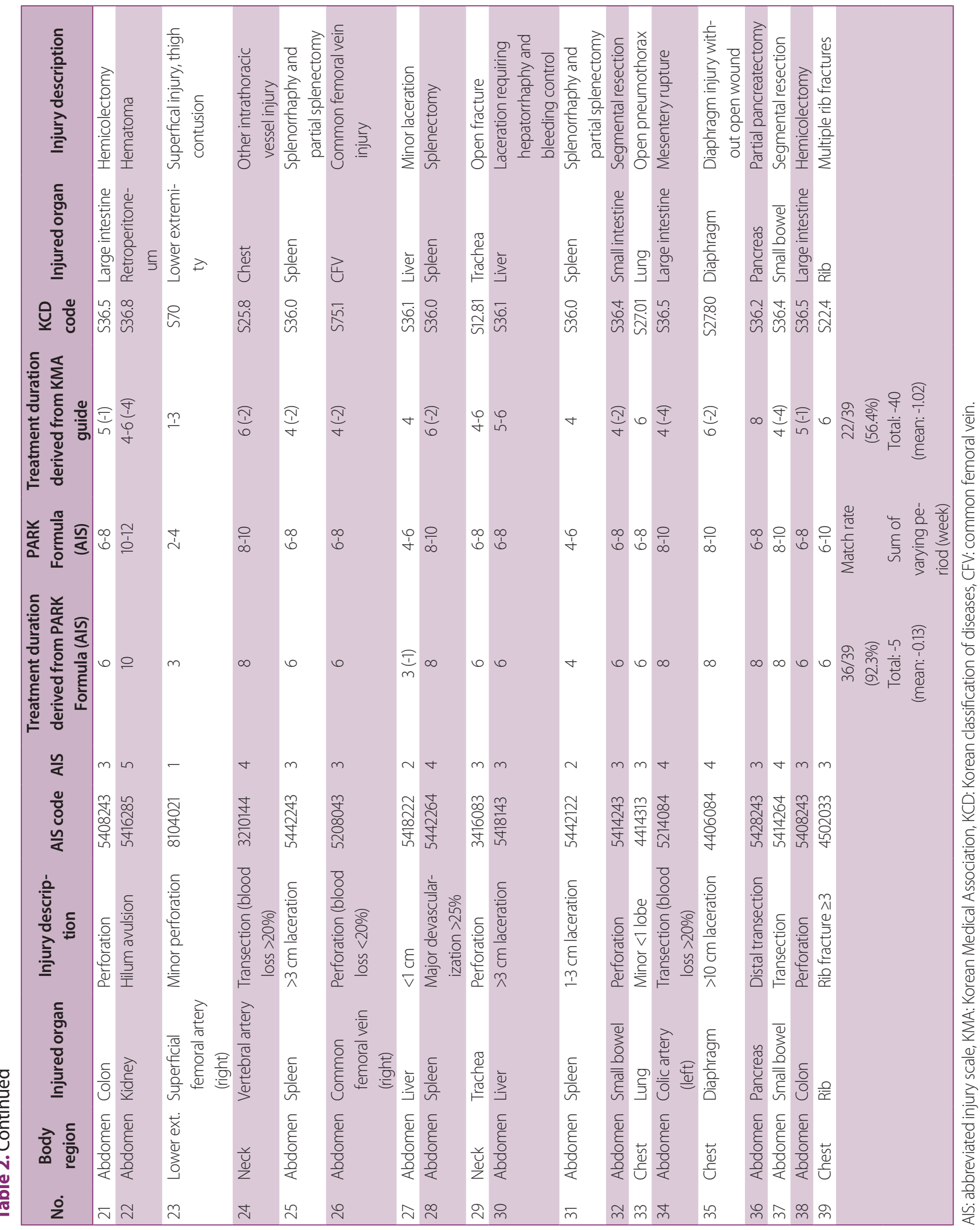


some cases extremely different between doctors and hospitals for the same organ injury with similar severity. These situation can cause distrust of their doctors, and in some cases, can result in legal issues, which makes appropriate decision of treatment duration of utmost importance $[1,7]$.

In the USA and Japan, there is no casualty medical certificate, there is only a medical certificate. Casualty medical certificate is a unique document in Republic of Korea. As the patients, judges, prosecutor, and insurance companies have no medical knowledge, they try to decide the severity of injury based on the treatment duration written in the casualty medical certificate. If the treatment duration is long, the injury is considered severe, and if the treatment duration is short, the injury is considered mild. This makes sense at a glance, but the truth is different. In general, severe injury requires more treatment duration, but there can be a difference according to which organ or tissue is injured. Also, the treatment duration can be different between similar injuries according to the treatment modality, and it also can be different according to the age and physical status of the patient. In consideration of these complexity, KMA published a guide to writing medical certificate containing 'treatment duration of each diagnosis in writing casualty medial certificate'. This standard guides uniform treatment duration regardless of the treatment modality. KMA acknowledges that this method has some limitations, but maintains this guideline for the reason that there is no alternative for this method [2-4]. On the other hand, some doctors utilize McBride's 'disability evaluation and principles of treatment of compensable injuries' to decide the treatment duration [8].

Kim and In [6] pointed out some problems with 'Guide to Medical Certificate issued by KMA' in the text 1) difference of treatment duration, 2) validity of treatment duration, 3) treatment duration according to age, 4) difference according to fracture severity (displacement, comminution, open fracture, etc.), and 5) treatment modality and real treatment duration. Also, Kim et al. [1] pointed out some problems such as 1) treatment duration according to the diagnosis of injury, 2) difference between clinical departments, 3) omission for the diagnosis of peripheral nerve injury, 4) error in the typing for printing, and 5) distribution of the booklet.

Authors tried to point out the problems related to 'treatment duration of each diagnosis in writing casualty medial certificate' of KMA and also to find out the solution to improve problems related to this. The problems are 1) There is no correlation between the severity of injury and the treatment duration. 2) KMA mingles diagnosis and operation name. For example, mild liver laceration, liver hematoma is used together with suture of liver parenchyma, liver laceration requiring suture, partial hepatectomy, liver lobectomy. This mingled use can also be found in other abdominal organ injury (Table 3). 3) Description of the injury is too much simplified in many cases. In KMA guide, abdominal injury is classified into 50 categories [4], but in AIS dictionary, it is classified into 250 categories [6]. And 4) The degree of injury is unified in KMA guide, but AIS dictionary has more detailed description of the degree of injury. For example, most severe form of liver injury is described simply as 'lobectomy' in the KMA guide, but the AIS dictionary describes this as 'parenchymal disruption of $>75 \%$ of hepatic lobe or $>3$ Couinaud's segments within a single lobe or involving retrohepatic vena cava/central hepatic veins or massive

Table 3. Treatment duration change of liver injury in Korean Medical Association (KMA) guide

\begin{tabular}{|lccc|}
\hline Injury description & Correlation to OIS & \multicolumn{2}{c|}{ Treatment duration (week) } \\
\cline { 3 - 4 } Minor laceration (laparotomy) & 1994 KMA guide & 2003, 2015 KMA guide \\
Intrahepatic hematoma & I & 3 & 4 \\
Laceration requiring hepatorrhaphy and bleeding control & II & $3-6$ & $4-6$ \\
Partial resection & N & 5 & $5-6$ \\
Lobectomy & V & 6 & $6-8$ \\
\hline
\end{tabular}

OIS: organ injury scale. 
or complex or (organ injury scale [OIS] V)', which shows much more detailed description (Table 4). If an injury is described as simple as KMA guide, it is difficult to find an appropriate description for the injury of the patient, and the doctor's subjective opinion has to be applied in many cases.

In order to improve these problems, PARK Formula (AIS) was developed utilizing AIS. PARK Formula (AIS) has close correlation between AIS, which is an ordinal scale related to the injury severity, and treatment duration. The authors referred KMA 2015 guideline to find the pattern between injury severity and treatment dura- tion. We adjusted the treatment duration of liver injury for OIS, and tried to find out a pattern between OIS grade and treatment duration. And then, a formula is derived utilizing AIS, and PARK Formula (AIS) is our result (Table 5).

In the case of a patient's specific organ injury, we can find appropriate injury from the AIS dictionary easily. And inputting the identified AIS score to the PARK Formula (AIS), we can calculate treatment duration which has 3 weeks of range. For example, AIS 1 is 2-4 weeks, AIS 2 is $4-6$ weeks, AIS 3 is 6-8 weeks, AIS 4 is $8-10$ weeks, and AIS 5 is $10-12$ weeks. Within each range, mild injury

Table 4. AIS code and injury description for liver injuries [9]

\begin{tabular}{|ll|}
\hline AIS 2005 & \multicolumn{1}{c|}{ Injury description } \\
\hline 541899.2 & Liver NFS \\
\hline 541810.2 & Contusion; hematoma NFS \\
\hline 541812.2 & Subcapsular, $\leq 50 \%$ surface area, or nonexpanding; intraparenchymal $\leq 10 \mathrm{~cm}$ in diameter; minor; superficial [OIS I, II] \\
\hline 541814.3 & Subcapsular, $>50 \%$ surface area or expanding; ruptured subcapsular or parenchymal; intraparenchymal $>10 \mathrm{~cm}$ or expanding; major [OIS III] \\
\hline 541820.2 & Laceration NFS \\
541822.2 & Simple capsular tears; $\leq 3 \mathrm{~cm}$ parenchymal depth; $\leq 10 \mathrm{~cm}$ long; minor; superficial [OIS II] \\
\hline 541824.3 & $>3$ cm parenchymal depth; major duct involvement; moderate [OIS III] \\
\hline 541826.4 & Parenchymal disruption $\leq 75 \%$ hepatic lobe; multiple lacerations $>3$ cm deep; "burst" injury; major [OIS IV] \\
\hline 541828.5 & Parenchymal disruption of $>75 \%$ of hepatic lobe or $>3$ Couinaud's segments within a single lobe; or involving retrohepatic vena cava/cen- \\
\hline 541830.6 & tral hepatic veins; massive; complex [OIS V] \\
\hline 541840.4 & Rupture \\
\hline
\end{tabular}

AIS: abbreviated injury scale, NFS: not further specified, OIS: organ injury scale.

Table 5. Calculation of PARK Formula (AIS) from 2015 KMA guidelines for medical certificates and AAST OIS

\begin{tabular}{|lccc|}
\hline \multirow{2}{*}{ AAST } & AAAM & \multicolumn{2}{c|}{ Treatment duration (week) } \\
\hline OIS & AIS & KMA 2015 guide & PARK Formula (AIS) \\
\hline I & 2 & KMA 2015 guide $>$ adjust to OIS & $($ AlS $\times 2) \sim([$ AIS $\times 2]+2)$ \\
II & 2 & $4-5$ & $4-6$ \\
III & 3 & $5-6$ & $4-6$ \\
IV & 4 & $6-8$ & $6-8$ \\
V & 5 & $6-12$ & $8-10$ \\
VI & 6 & None & $10-12$ \\
\hline
\end{tabular}

AIS: abbreviated injury scale, KMA: Korean Medical Association, AAST: American Association for the Surgery of Trauma, OIS: organ injury scale, AAAM: Association for the Advancement of Automotive Medicine.

When OIS is applied instead of AIS. 
belongs to the lowest value, moderate injury belongs to middle value, and severe injury belongs to the highest value within the range. Also, for solid organ injury, even if the discrimination of AIS is in paucity, we can easily calculate the treatment duration without difficulty in PARK Formula (AIS). This is because the treatment duration of 1 level low severe injury has the same value with 1 level high mild injury. For example, AIS 2 with severe injury has the same treatment duration with AIS 3 with mild injury which is 6 weeks.

The authors were able to easily utilize PARK Formula (AIS) in writing casualty medical certificate with the use of AIS which is very familiar to trauma surgeons to decide treatment duration. In this study, 36 out of 39 cases (92.3\%) were matched in actual treatment duration of casualty medical certificate and the treatment duration calculated by PARK Formula (AIS). The other three patients who showed difference in treatment duration, one patient required 1 week less treatment duration, and two patients required 2 weeks less treatment duration. The case which required 1 week less treatment duration was liver injury with AIS 2 (OIS I). In American Association for the Surgery of Trauma OIS, liver injury has separate OIS I and OIS II (Table 6). This is also the same in other solid organ such as spleen, kidney, and pancreas. However, AIS dictionary classifies these two OIS to AIS 2 (no AIS 1 for liver injury), and this is the reason for this difference.
Therefore, in the case of solid organ injury of AIS 2 (OIS I), it may need to consider applying AIS 1 in calculating treatment duration. Of the cases which required 2 weeks less treatment duration, one case was AIS 3 small bowel injury (perforation) which the author decided wrong as AIS 2, and the other case was 4 years old patient AIS 4 spleen injury which was underestimated because of the extremely small size of the spleen compared to the adult.

Comparing the treatment duration result between KMA guideline and PARK Formula (AIS), there were 17 discrepancy cases. Two cases showing 1 week less treatment duration were all colon perforation, and 11 cases (64.7\%) showing 2 weeks less treatment duration were one AIS 3 common femoral vein injury, two AIS 3 small bowel injury, two AIS 3 spleen injury, four AIS 4 spleen injury, one lumbar artery injury, and one diaphragm injury. Four cases showing 4 weeks less treatment duration were one AIS 4 left colic artery injury, one AIS 4 small bowel injury, one AIS 5 spleen injury, and one AIS 5 kidney injury.

Compared with PARK Formula (AIS), the treatment duration of KMA guide was generally short, but the difference was 1.02 weeks which was not great. There should be discussion in the trauma specialist society about this issue. Also, for the difference between clinical departments, there should be discussion between departments. For a good consensus to be made, KMA should mediate in a very wise way. Also in making a consensus, PARK

Table 6. AAST OIS for liver injuries [10]

\begin{tabular}{|c|c|c|}
\hline \multicolumn{2}{|c|}{ Grade } & \multirow[t]{2}{*}{ Injury description } \\
\hline I & Hematoma & \\
\hline & Laceration & Capsular tear, $<1 \mathrm{~cm}$ parenchymal depth \\
\hline \multirow[t]{2}{*}{$\|$} & Hematoma & $\begin{array}{l}\text { Subcapsular, } 10-50 \% \text { surface area } \\
\text { Intraparenchymal, }<10 \mathrm{~cm} \text { diameter }\end{array}$ \\
\hline & Laceration & $1-3 \mathrm{~cm}$ parenchymal depth, $<10 \mathrm{~cm}$ length \\
\hline \multirow[t]{2}{*}{ III } & Hematoma & $\begin{array}{l}\text { Subcapsular, }>50 \% \text { surface area or expanding. Ruptured subcapsular or parenchymal hematoma } \\
\text { Intraparenchymal hematoma }>10 \mathrm{~cm} \text { or expanding }\end{array}$ \\
\hline & Laceration & $>3 \mathrm{~cm}$ parenchymal depth \\
\hline IV & Laceration & Parenchymal disruption involving $25-75 \%$ of hepatic lobe or $1-3$ Coinaud's segments in a single lobe \\
\hline \multirow[t]{2}{*}{ V } & Laceration & Parenchymal disruption involving $>75 \%$ of hepatic lobe or $>3$ Coinaud's segments within a single loge \\
\hline & Vascular & Juxtahepatic venous injuries ie. Retrohepatic venal cava/central major hepatic veins \\
\hline IV & Vascular & Hepatic avulsion \\
\hline
\end{tabular}

AAST: American Association for the Surgery of Trauma, OIS: organ injury scale. 
Formula (AIS) can be a great help in solving the various problems with KMA guide.

$$
\text { PARK Formula }\left(\text { AIS }^{\star}\right)=\left(\text { AIS }^{\star} \times 2\right) \sim\left(\left[\text { AIS }^{\star} \times 2\right]+2\right)
$$

Applying this formula uniformly to all situations for deciding treatment duration may be unreasonable, and this equation can give a value that is too high or too low compared to the expert opinion. To solve this problem, we can use PARK Formula (AIS*), an upgraded version of PARK Formula (AIS). PARK Formula (AIS*) uses AIS in a modified way, which add or subtract weighted value of 0.5 discrimination according to the agreement of expert opinion, which we express as AIS*. For example, if treatment duration of 4-6 weeks for AIS 2 calculated by PARK Formula (AIS) is considered too high, we can modify the AIS subtracting 0.5 (a value of 1.5 which is AIS*), and the treatment duration can be reduced to 3-5 weeks. In the same case, if the treatment duration of 5-7 weeks is more appropriate, 0.5 can be added to the AIS (a value of 2.5 which is $\mathrm{AIS}^{*}$ ), and the treatment duration can be increased to 5-7 weeks.

$$
\text { PARK Formula }\left(\text { AIS }^{\star} \alpha\right)=\left(\text { AIS }^{\star} \times 2\right) \sim\left(\left[\text { AIS }^{\star} \times 2\right]+[2+\alpha]\right)
$$

Using both PARK Formula (AIS) and PARK Formula $\left(\right.$ AIS $\left.^{*}\right)$, the treatment duration is decided within 3 weeks of range. In case where more than 3 weeks of range is needed, $\alpha$ value can be increased with value 1 discrimination as needed. For example, if the result by PARK Formula $\left(\mathrm{AIS}^{*}\right)$ is $5-7$ weeks but $5-10$ weeks is more adequate, $\alpha$ value can be adjusted to 3 to make the corresponding range.

\section{CONCLUSION}

For the decision of the treatment duration in trauma patients, utilizing worldwide used AIS scoring system is very efficient. Using PARK Formula (AIS), doctors can document casualty medical certificate with ease. Also, this can free from legal problem that doctors can be involved. KMA should provide more practical 'treatment duration of each diagnosis in writing casualty medial certificate' for the doctors. PARK Formula (AIS) can be a good alternative for this effort.

\section{REFERENCES}

1. Kim BH, Im JI, Im YK, Nam U. Review of guide to medical certificate issued by Korean Medical Association. J Korean Soc Fract 1999;12:56-60.

2. Korean Medical Association. Guidelines for medical certificates. Seoul:Korean Medical Association;1996.

3. Korean Medical Association. Guidelines for medical certificates. Seoul:Korean Medical Association;2003.

4. Korean Medical Association. How to Write and Issue Medical Certificates. Seoul:Korean Medical Association;2015.

5. Bae H. Physician liability and social responsibility related with medical certificates. Ewha Med J 2013;36:101-11.

6. Kim BH, In YK. Medical certificate in orthopedic trauma patient: reasonable duration of expected treatment. J Korean Soc Fract 1995;8:675-7.

7. McBride ED. Disability evaluation and principles of treatment of compensable injuries. 6th ed. Philadelphia:B. Lippincott Company;1963.

8. Kim DS. The social problems surrounding doctor's certificates. J Korean Med Assoc 2014;57:580-8.

9. Association for the Advancement of Automotive Medicine. The Abbreviated Injury Scale 2015 Revision. Chicago:Association for the Advancement of Automotive Medicine;2016.

10. Moore EE, Cogbill TH, Jurkovich GJ, Shackford SR, Malangoni MA, Champion HR. Organ injury scaling: spleen and liver (1994 revision). J Trauma 1995;38:323-4. 